Federica Masiero

\title{
Ist ein älterer Herr un signore anziano oder un signore piuttosto anziano?
}

\section{Über die Wiedergabe des absoluten Komparativs im Italienischen}

\begin{abstract}
The present paper is concerned with the translation of the German absolute comparative (ein größerer Beitrag, ein älterer Herr) into Italian. In contrast to German the Italian comparative form does not perform any absolute function. The central aim of this paper consists on the one hand in presenting the different possibilities available in Italian of translating the German absolute comparative on the basis of a corpus of literary texts. On the other hand, the commonly occurring translating difficulties are analysed.
\end{abstract}

Schlagwörter: absoluter Komparativ, Übersetzung, Deutsch, Italienisch

DOI 10.1515/les-2015-0002

\section{Das Phänomen}

Die sprachliche Erscheinung des absoluten Komparativs wird in den deutschen Grammatiken größtenteils durch die lexikalisierte absolute Komparativform älter veranschaulicht wie im folgenden Beispiel:

(1) Ein älterer Lehrer saß im Raum.

In (1) drückt die Steigerungsform älter einen niedrigeren bzw. geringeren Grad von alt aus. Der Lehrer ist über das mittlere Lebensalter hinaus, nicht mehr jung, aber auch noch nicht ganz alt: Er ist noch etwas jünger als ein alter Lehrer. Solche Komparativstrukturen stellen Verwendungen dar, bei denen die Komparationsbasis nicht erwähnt wird. Sie unterscheiden sich von den Vergleichskonstruktionen, bei denen die Vergleichsbasis deutlich genannt wird wie in (2):

Federica Masiero: Dipartimento di Studi linguistici e letterari, Via B. Pellegrino, 26, Università degli Studi di Padova, E-Mail: federica.masiero@unipd.it 
(2) Karin ist älter als Anna.

bzw. von den Vergleichskonstruktionen, bei denen sie vorerwähnt ist wie in (3):

(3) Marx hat Wein gekauft. Whisky wäre besser gewesen.

sowie von denen, bei denen die Vergleichsbasis aufgrund des gemeinsamen Hintergrundwissens erschlossen werden kann wie in (4):

(4) 2016 werden Gas und Wasser teurer.

Die in (2)-(4) veranschaulichten Komparativformen werden auch relativer Komparativ genannt. Mit den bisher benutzten Termini absoluter Komparativ und relativer Komparativ schließe ich mich an die Terminologie an, die Ulrich Engel in seiner Grammatik (1988:568-569; ebenso 2004:344-346) vorschlägt.

Der absolute Komparativ in (1) drückt, wie oben vermerkt, keine Steigerung der durch den Positiv bezeichneten Eigenschaft aus, sondern eine Abschwächung. Dies wird meistenteils (s. Schefer-Krayenbühl 1989:53, Thurmair 2001:228f., Albers 2007:15-20; anders bei Engel 1988:569, 2004:570) anhand von Bewertungsskalen veranschaulicht. Im Falle des absoluten Komparativs werden die Bewertungsskalen durch ein antonymisches Paar graduierbarer Adjektive aufgespannt. Das sei exemplarisch anhand der absoluten Komparativformen länger (Positiv: lang) und kürzer gezeigt. Bei länger und kürzer ist die Bewertungsskala wie folgt abzubilden:

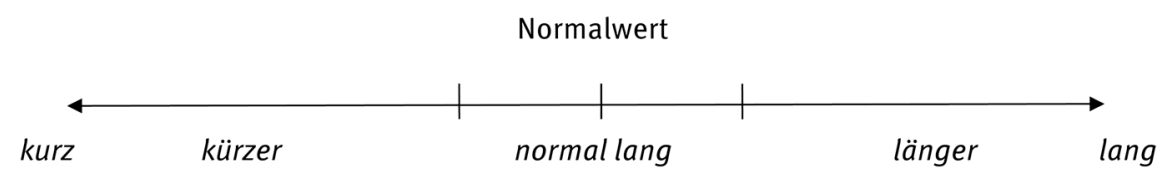

Abb. 1

Wie die Graphik verdeutlicht, ist der Geltungsbereich des absoluten Komparativs länger links von der Positivform lang zu verorten. Eine längere Reise ist deshalb kürzer als eine lange Reise: Die Reise ist nicht kurz, aber noch nicht ganz lang. Dabei darf nicht übersehen werden, dass die Abschwächung der Eigenschaftsaussage des Positivs im absoluten Komparativ von dem Zielantonym (lang) in Richtung des Ausgangsantonyms (kurz) regressiv, von dem Ausgangsantonym (kurz) in Richtung des Zielantonyms (lang) aber progressiv erfolgt (s. Trost 2006:40f.). Während eine längere Reise kürzer als eine lange Reise ist, ist eine 
kürzere Reise länger als eine kurze Reise, aber kürzer als eine längere Reise. Um zu wissen, was kürzer bedeutet, muss man aber wissen, was länger bedeutet und umgekehrt. Um dieses Problem zu lösen sind beide Formen auf einen Normalwert zu beziehen, der zwischen den beiden durch die jeweiligen Positive bezeichneten Skalenabschnitten zu verorten ist (s. Becker 2005:107-111). Dieser Bereich teilt die Eigenschaftsskala in zwei Hälften und stellt den Übergang von den Werten, die dem einen Pol zuzuordnen sind, zu denen, die dem anderen Pol zugehören, dar. Eine kürzere Reise ist deshalb kürzer als eine normal lange Reise, eine längere Reise ist länger als eine normal lange Reise. ${ }^{1}$

Aus dem oben Genannten soll deutlich geworden sein, dass der absolute Komparativ einen größeren Bedeutungsraum eröffnet als der Positiv. Dadurch wird der Aussagewert des absoluten Komparativs aber auch unbestimmter als der des Positivs.

Da sich die vorliegende Untersuchung mit der Wiedergabe des absoluten Komparativs befasst, soll hier diese kurze Charakterisierung des Phänomens ausreichen. $\mathrm{Zu}$ einer eingehenden Darstellung verweise ich auf die Arbeit von Becker (2005) und Albers (2007).

\section{Terminologie, Stand der Forschung und Fragestellung}

Ein Überblick über die gängigen deutschen Grammatiken, die dieses Phänomen behandeln, ${ }^{2}$ zeigt, dass die meisten darauf verzichten, den absoluten Komparativ vom relativen terminologisch abzugrenzen. Sie präsentieren das Phänomen als besondere Komparativverwendung, ohne eine terminologische Unterscheidung vorzunehmen. ${ }^{3}$ So schreibt z. B. die Grammatik von Jung Walter (1980:306):

Es gibt auch einen Komparativ ohne tatsächlichen Vergleich. Es bezieht sich dem Sinne nach auf ein gegensätzliches Adjektiv. Ein älterer Herr ist kein alter Herr, sondern jünger als ein alter Herr; eine längere Reise ist kürzer als eine lange Reise. [...] Diese Art des Komparativs kann auch adverbial verwendet werden.

1 Die Bestimmung des Normalwertes ist ein komplexer Vorgang und erfolgt je nach Sachgebiet unterschiedlich. Vgl. die Beobachtungen bei Trost (2005:108-112) und bei Albers (2007:16f.), die aber Trost nicht berücksichtigt.

2 Zu den Grammatiken, die dieses Phänomen nicht ansprechen, zählen u.a. Paul (1916-1920), Zifonun et al. (1997), Eisenberg (2004) und Wellmann (2008).

3 Einen repräsentativeren Überblick über die Behandlung des absoluten Komparativs in den deutschen Grammatiken auch in diachroner Perspektive bietet Becker (2005:100-110). 
Ebenso weisen Hentschel/Weydt (2013:150) darauf hin, dass der Komparativ keineswegs immer einen höheren Grad bedeutet, ohne aber das Phänomen terminologisch festzulegen: „Die Steigerung eines Adjektivs bzw. eines Adverbs bedeutet nicht unbedingt, dass das Maß der angegebenen Eigenschaft gesteigert wird, sondern kann in einigen Fällen auch eine Steigerung gegenüber dem jeweiligen Antonym ausdrücken.“

In der einschlägigen Forschungsliteratur sind Termini für die sprachliche Erscheinung des absoluten Komparativs zwar präsent. Es herrscht hier aber keine einheitliche Terminologie. Neben absoluter Komparativ (s. Engel 1998:568-569) sind Termini zu finden wie Inversionskomparativ (s. Renicke 1955:74), Normkomparativ (s. Weinrich u.a. 1987:120f.), Abschwächungs- oder Deminutionskomparativ (s. Trost 2006:37) sowie indifferenter Komparativ (s. Thurmair 2001:223). Die nicht einheitliche Terminologie widerspiegelt die unterschiedlichen Auffassungen, die in der Literatur über den absoluten Komparativ vertreten sind. Einen Überblick darüber liefern Becker (2005:100-107) und Albers (2007:27-55). Beckers Ausführungen erweisen sich als besonders geeignet, den Stand der Forschung zum absoluten Komparativ nachzuvollziehen. Becker ermittelt in der bisherigen Forschungsliteratur drei vorherrschende Beschreibungsansätze. Dabei unterscheidet er zwischen zwei dualistischen Theorien und einer monistischen Theorie. Die dualistischen (Engel und die Anhänger der sogenannten Antonymensteigerung) postulieren zwei Arten des Komparativs; die monistische (Weinrich) reduziert den absoluten Komparativ auf den relativen. Beckers (2005:107-112) eigener Beitrag zur Erforschung des Phänomens besteht darin, die dualistischen Theorien als unzureichend $\mathrm{zu}$ charakterisieren und die monistische $\mathrm{zu}$ vervollständigen. Hiernach wird nicht das Antonym, sondern ein postulierter Normalwert des Adjektivs als Vergleichswert herangezogen, zu dem dann wiederum der Komparativ in Beziehung gesetzt wird.

Wie aus dem oben Gesagten hervorgeht, liegt der Fokus der bisherigen Arbeiten vor allem auf theoretischen Aspekten, die vornehmlich die Verhältnisse zwischen absolutem und relativem Komparativ abzuklären versuchen und die auf eine theoretische Einordnung des Phänomens abzielen. Was den kontrastiven Gesichtspunkt anbelangt, so gibt es m.W. nur Untersuchungen zum Vergleich Deutsch-Englisch. Dabei seien die eingehendere vergleichende Studie von Ursula Schefer-Krayenbühl (1989) und der kurze Aufsatz von Friedrich (1994) erwähnt. Die geringe Anzahl der Analysen mit kontrastiver Sicht hängt sicherlich damit zusammen, dass der absolute Komparativ relativ selten ist. Außer in den klassischen Sprachen und im Deutschen ist der absolute Komparativ laut Becker (2005:99f.) nämlich nur im britischen und amerikanischen Englisch sowie im Tschechischen vorhanden, wobei er in den genannten Sprachen mit unterschiedlicher Häufigkeit auftritt. 
Es fehlen bisher Arbeiten, die sich eingehend mit der Entstehung des absoluten Komparativs auseinandersetzen, worauf Becker (2005:99) hinweist, sowie Arbeiten, die die tatsächliche Wiedergabe des deutschen absoluten Komparativs in Sprachen, die das Phänomen nicht aufweisen, in den Vordergrund stellen. Über dieses Thema fügt lediglich Janine Albers (2007:108-110) in ihre Arbeit einen kurzen Exkurs ein. Darin skizziert sie exemplarisch anhand des Spanischen und des Französischen, die den Komparativ in absoluter Form nicht verwenden, die alternativen Ausdrucksmöglichkeiten dafür. Dabei bezieht sich die Autorin auf vergleichende Grammatiken Spanisch-Deutsch bzw. Französisch-Deutsch. Das Italienische, das wie die von Albers untersuchten Sprachen keinen Komparativ in absoluter Form aufweist, wird nicht berücksichtigt.

Die vorliegende Studie versucht, diese Forschungslücke zumindest teilweise zu schließen, indem die Wiedergabe des deutschen absoluten Komparativs im Italienischen untersucht werden soll. Dies erfolgt anhand einer empirischen Analyse von konkreten Beispielen aus literarischen Texten. Somit soll an empirischem Material dargestellt werden, welche Übersetzungsvarianten eingesetzt werden, um den deutschen absoluten Komparativ im Italienischen wiederzugeben, und inwieweit diese $\mathrm{zu}$ einer adäquaten Übersetzung führen. Anhand dieser Daten sollen die häufigsten Übersetzungsprobleme identifiziert werden. Aus den Beobachtungen werden dann generalisierende Implikationen abgeleitet.

Bevor wir uns den Übersetzungen zuwenden, soll kurz illustriert werden, wie der absolute Komparativ in den für das italienische Publikum geschriebenen Fremdsprachengrammatiken ${ }^{4}$ des Deutschen, in den für das deutsche Publikum geschriebenen Fremdsprachengrammatiken des Italienischen sowie in Studien, die eine kontrastive Analyse Italienisch-Deutsch durchführen, behandelt wird. Ein solcher Überblick soll zeigen, ob bzw. inwieweit Grammatiken und kontrastive Analysen Wesen und Gebrauch des absoluten Komparativs ansprechen, ob sie Vorschläge für die Übersetzung ins Italienische anbieten und ob Übersetzungen, die diesen Vorschlägen entsprechen, in dem zu untersuchenden Korpus vorzufinden sind. Anschließend soll des Weiteren ein kurzer Blick auf die Behandlung der gebräuchlichsten absoluten Komparativformen sowohl in DaF-Wörterbüchern als auch in zweisprachigen Wörterbüchern Deutsch-Italienisch/Italienisch-Deutsch geworfen werden.

4 Der Terminus Fremdsprachengrammatik ist hier im Sinne von Kleineidam/Raupach (1995:300) zu verstehen. Damit ist gemeint: „eine kursunabhängige Beschreibung der Regularitäten der FS [=Fremdsprache], welche dem FS-Lerner zum orientierenden Nachschlagen und zum wiederholenden Lernen dienen soll und gegebenenfalls zur planvollen Erweiterung des Gelernten“. 


\section{Der absolute Komparativ in Grammatiken, in kontrastiven Studien und Wörterbüchern}

In den für das italienische Publikum geschriebenen Fremdsprachengrammatiken des Deutschen wird selbstverständlich der relative Komparativ behandelt. Von den insgesamt 19 herangezogenen Grammatiken ${ }^{5}$ sind es aber nur fünf, die das Phänomen des absoluten Komparativs ansprechen. Dabei handelt es sich um folgende Grammatiken: Bornmann/Mauser (1964:246), Azaïs (1994:67), Seiffarth/ Medaglia (1998:133), Difino/Fornaciari (1999:266) und Weerning/Mondello (1996/ 2000: §51). Bei der Behandlung des Phänomens weisen diese Grammatiken einige charakteristische Merkmale auf, die wie folgt zusammenzufassen sind:

- In allen fünf Grammatiken wird der absolute Komparativ im Rahmen der Ausführungen zur Komparation als Spezialfall kurz abgehandelt. Bis auf Azaïs (1994:67) wird auf eine spezifische Terminologie verzichtet, Azaïs spricht vom falschen Komparativ.

- Die abschwächende Funktion des absoluten Komparativs im Verhältnis zum Positiv wird entweder gar nicht benannt (Azaiis 1994:67) oder vage gefasst (s. Weerning/Mondello 1996/2000: § 51; Seiffarth/Medaglia 1998:113). Weerning/Mondello führen Folgendes aus: "Il comparativo si può usare a volte senza termine di paragone per moderare un'affermazione o dare una valutazione prudente” (Der Komparativ kann manchmal ohne Vergleichsbasis verwendet werden, um eine Aussage $\mathrm{zu}$ mildern oder um eine vorsichtige Wertung zu geben). Seiffarth/Medaglia verweisen nur auf die Verwendung des absoluten Komparativs für eine vorsichtige und höfliche Charakterisierung: "alcune forme del comparativo vengono impiegate per esprimere un significato equivalente ma in forma meno cruda” (einige Komparativformen werden verwendet, um eine äquivalente Bedeutung [wie die des relativen Komparativs] auszudrücken, aber in höflicherer Weise). Difino/Fornaciari (1999:266) scheinen dem absoluten Komparativ sogar eine verstärkende Funktion im Verhältnis zum Positiv beizumessen: “[...] il comparativo di maggioranza senza il secondo termine di paragone si usa in luogo di un aggettivo di grado positivo per rafforzarne il significato” (der Steigerungskomparativ ohne Vergleichsbasis wird anstatt des Positivs gebraucht, um dessen Bedeutung zu verstärken). Die einzige Grammatik, die eindeutig die abschwächende Funktion des absoluten Komparativs im Vergleich zum Po-

5 Die in dieser Untersuchung herangezogenen Grammatiken sind in der Bibliografie unter „Deutsche Fremdsprachengrammatiken für Italiener“ aufgelistet. 
sitiv anspricht, ist die Grammatik von Bornmann/Mauser (1964:246): “[...] essi servono ad attenuare il significato dell' aggettivo" (sie [die absoluten Komparativformen] dienen dazu, die Bedeutung des Adjektivs abzuschwächen).

- Welche Möglichkeiten der Übersetzung ins Italienische es gibt, muss in der Regel den spärlichen angeführten Beispielen entnommen werden. Generalisierende, zusammenfassende Ausführungen fehlen nahezu immer. Die einzige Ausnahme bilden diesbezüglich Difino/Fornaciari (1999:266), die Folgendes ausführen: "In italiano lo si renderà mediante avverbi quali alquanto, piuttosto + aggettivo, oppure ricorrendo ad aggettivi più appropriati” (Im Italienischen wird er [der absolute Komparativ] mit Adverbien wie alquanto, piuttosto + Adjektiv oder mit geeigneteren Adjektiven wiedergegeben). Dass der absolute Komparativ im Italienischen meistens mit einer Periphrase (älter: piuttosto anziano, piuttosto vecchio, non più tanto giovane, di una certa età; jünger: ancora giovane, piuttosto giovane; länger: alquanto a lungo, piuttosto lungo), in bestimmten Fällen aber auch mit einem Einzelwort wiedergegeben werden kann (älter: anziano; größer: cospicuo, considerevole, rilevante, certo/a; kleiner: minori), ${ }^{6}$ ist den in den Grammatiken angeführten Beispielen nicht immer eindeutig zu entnehmen. ${ }^{7}$ Für ein und dieselbe absolute Komparativform finden sich zudem auch unterschiedliche Übersetzungsvorschläge: Ist ein älterer Herr bzw. eine ältere Dame un signore anziano bzw. una signora anziana (s. Difino/Formaciari 1999:266) oder un signore piuttosto anziano bzw. una signora piuttosto anziana (s. Weerning/Mondello 1996/ 2000: $§ 51)$ ? Inwieweit diese Übersetzungen angemessen bzw. nicht angemessen sind, wird in 5 . erörtert.

Zusammenfassend kann man sagen, dass das Thema des absoluten Komparativs in den untersuchten Grammatiken nicht scharf genug gefasst wird. Es wird nicht genug deutlich gemacht, dass der Komparativ im Italienischen in absoluter Funktion nicht verwendet wird, und es wird ferner nicht hinlänglich beschrieben, welche Bedeutung der absolute Komparativ im Vergleich zum Positiv hat. Diese beiden Aspekte stellen aber ein zentrales Wissen für eine angemessene Übersetzung ins Italienische sowie für ein korrektes Verständnis der angebotenen Übersetzungsvorschläge dar.

6 Die in Klammern genannten Übersetzungsmöglichkeiten stammen aus den fünf untersuchten Grammatiken.

7 Das hängt hauptsächlich von der Anzahl der angeführten Beispiele ab. Falls die Anzahl sehr gering ist (s. Seiffarth / Medaglia und Azaïs), ist der Überblick über die möglichen Wiedergaben im Italienischen unbefriedigend. 
In den auf Deutsch verfassten Fremdsprachengrammatiken des Italienischen wird der absolute Komparativ äußerst selten angesprochen. Christoph Schwarze, der sich in seiner Grammatik (1988:592-612) ausführlich mit dem Thema Vergleiche befasst, nennt das Phänomen nicht. Unter den berücksichtigten Grammatiken ${ }^{8}$ sprechen lediglich Reumuth/Winkelmann (2011:205) das Phänomen an. Im Rahmen ihrer Darstellung zum Komparativ finden sich in einer Anmerkung die folgenden Ausführungen:

In folgenden Ausdrücken wird im Italienischen kein Komparativ verwendet: ein älterer Herr un signore piuttosto anziano/di una certa età, die älteren Leute gli anziani, die jüngere/ältere Generation la nuova/vecchia generazione, Plinius der Ältere Plinio il Vecchio, meine bessere Hälfte la mia dolce metà, eine längere Reise un viaggio un po' lungo, im engeren/weiteren Sinn in senso stretto/largo, die neueren Sprachen le lingua moderne, ein kleineres/größeres Erdbeben una leggera/pesante scossa di terremoto. ${ }^{9}$

Auch hier finden sich inkonsequente Übersetzungsvorschläge: Warum ist z.B. eine längere Reise un viaggio un po' lungo (eine etwas lange Reise), aber ein größeres Erdbeben una pesante scossa di terremoto (ein starkes Erdbeben) und nicht z.B. una scossa di terremoto piuttosto pesante (vgl. zur Angemessenheit dieses Übersetzungsvorschlages 5.2.h)? Reumuth/Winkelmann verzichten sowohl auf eindeutige Übersetzungsrichtlinien als auch auf eine spezifische Terminologie für das Phänomen. Was den terminologischen Aspekt anbelangt, präsentieren sich auf Deutsch verfasste Fremdsprachengrammatiken des Französischen und Spanischen, die wie das Italienische keinen absoluten Komparativ aufweisen, besser. Dethloff/Wagner (2007:64) verwenden in ihrer Grammatik des Französischen für den deutschen absoluten Komparativ den Terminus der absolute Komparativ/le comparatif absolu, Cartagena/Gauner (1989:I, 354) sprechen in ihrer Grammatik des Spanischen von dem abschwächenden Komparativ.

Von den Gesamtdarstellungen, die eine kontrastive Analyse ItalienischDeutsch durchführen, wird das Phänomen nicht angesprochen. Die folgenden Gesamtdarstellungen wurden konsultiert: Figge/Matteis (1982), Blasco Ferrer (1999), Bosco Coletsos/Costa (2004), Catalani (2004), Nied Curcio (2008) und Cinato Kather (2011).

Was die Behandlung der Komparativformen in DaF-Wörterbüchern betrifft, so lexikalisiert unter den untersuchten DaF-Wörterbüchern das Langenscheidt

\footnotetext{
8 Die berücksichtigten Fremdsprachengrammatiken sind in der Bibliografie unter „Italienische Fremdsprachengrammatiken für Deutsche“ aufgelistet.

9 In dieser Breite nur in der Neubearbeitung 2011. In den früheren Auflagen (seit 1989: § 166) waren die Ausführungen deutlich knapper: „Merke: ein älterer Herr - un signore piuttosto anziano / di una certa età.“
} 
Großwörterbuch Deutsch als Fremdsprache die größte Anzahl Komparativformen. ${ }^{10}$ In der Erstauflage (1993) haben zunächst die absoluten Komparativformen länger und öfter(s) einen Sonderstatus, indem sie mit einer eigenen Eintragung bedacht werden. Ab 2003 fügt sich die Form älter hinzu. Pons Großwörterbuch DaF (2004) (2006) (2011) führt nur die Form öfter(s) auf. Keine lexikalisierte Komparativform weisen folgende Wörterbücher auf: Wörterbuch DaF De Gruyter (2000), Duden - Deutsch als Fremdsprache (2002) (2010), Wahrig Großwörterbuch $\operatorname{DaF}(2008)$.

In den gängigen zweisprachigen Wörterbüchern Deutsch-Italienisch / Italienisch-Deutsch ist öfter(s) unter den in der deutschen einsprachigen Lexikographie als eigene Lemmata geführten Komparativformen (s. Fußnote 10) die einzige, die schon seit den 60er Jahren mit einer eigenen Eintragung aufgeführt wird (s. Bidoli/Cosciani 1957, Ciardi/Dupré 1958). Die Formen jünger, kürzer, länger sind in der Lexikographie der 60er Jahre sonst meistens nur unter dem Lemma der Positivform zu finden. Ab den 80er Jahren führt das Wörterbuch Sansoni alle obengenannten absoluten Komparativformen als eigene Lemmata. Pons/Zanichelli (2001) (2007) weisen nur die Formen älter und öfter(s) auf. Auch in der Auflage 2009 ist länger nur unter lang zu finden.

\section{Charakteristika des Korpus und zu untersuchende Adjektive}

Bei dem zu untersuchenden Korpus handelt es sich ausschließlich um literarische Texte, und zwar Romane und Novellen. Durch die Begrenzung auf fiktionale narrative Texte bleibt das Korpus weitgehend einheitlich. Insgesamt werden der Untersuchung Werke aus den 20er bis zu den 60er Jahren des 20. Jahrhunderts zugrunde gelegt. Die relativ breite zeitliche Spanne im Korpus ist nicht relevant, da das Phänomen des absoluten Komparativs diachron keine Veränderung erfährt (s. Schefer-Krayenbühl 1989:126-132). Die Quellenauswahl sichert den Zugriff auf ein reichhaltiges Belegkorpus, da die ausgewählten Autoren einen reichen Gebrauch von absoluten Komparativformen machen. Bei den ausgewähl-

10 Die DaF-Wörterbücher wurden auf die absoluten Komparativformen hin untersucht, die in der einsprachigen deutschen Lexikographie als eigenes Lemma geführt werden, d.h.: älter, jünger, kürzer, länger, öfter(s). Vgl. dazu Trost (2006:39). Trost bezieht sich in seiner Untersuchung auf Duden - Universalwörterbuch (1989 und folgende Auflagen). Wahrig (2000 und folgende Auflagen) lexikalisiert weniger Komparativformen als Duden. Es fehlt z.B. die Form länger, die unter dem Lemma der Positivform erscheint. 
ten Autoren handelt es sich um Thomas Mann, Franz Kafka und Günter Grass. Von ihnen wurden folgende Werke und folgende italienische Übersetzungen ausgewertet:

Thomas Mann (1912): Der Tod in Venedig

La morte a Venezia (1970), Übersetzung von Emilio Castellani

La morte a Venezia (2004), Übersetzung von Anita Rho

La morte a Venezia (2010), Übersetzung von Emilio Castellani und neu durchgesehen von Renata Colorni

ders. Der Zauberberg

La montagna incantata (1965), Übersetzung von Ervino Pocar

La montagna magica (2010), Übersetzung von Renata Colorni

ders. (1954): Bekenntnisse des Hochstaplers Felix Krull

Confessioni del cavaliere d'industria Felix Krull (1965), Übersetzung von

Lavinia Mazzucchetti

Franz Kafka (1925): Der Prozeß

Il processo (1975), Übersetzung von Ervino Pocar

Il processo (1983), Übersetzung von Primo Levi

Il processo (1989), Übersetzung von Giuseppe Landolfi Petrone e Maria

Martorelli

Il processo (1995), Übersetzung von Anita Raja

Günther Grass (1959): Die Blechtrommel

Il tamburo di latta (1962), Übersetzung von Lia Secci

ders. (1974): Katz und Maus

Gatto e topo (2009), Übersetzung von Enrico Filippini

Nach der Beschreibung des Korpus besteht der zweite Schritt unserer Untersuchung in der Identifizierung der absoluten Komparativformen im Korpus. Bei der Identifizierung der absoluten Komparativformen im Korpus bediene ich mich des Ausschlussverfahrens nach Albers (2007). Albers entwickelt in ihrer Arbeit Kriterien, die dazu dienen, unter den in einem Korpus vorhandenen Komparativformen die relativen zu identifizieren. Durch deren sukzessiven Ausschluss werden die absoluten ermittelt. Albers' Kriterien zur Bestimmung der relativen Komparativformen sind von zweierlei Art: satzbezogen und kontextbezogen. Unter den satzbezogenen Kriterien führt sie folgende auf (2007:64):

a) Vorkommen einer Vergleichspartikel (als, wie, denn)

b) Vorkommen einer quantifizierenden Bestimmung (je...desto-Konstruktionen und Ergänzungen wie 2,5 cm)

c) Nennung des zweiten Vergleichselements im Satz

d) noch + Komparativ 
e) je(mals)/kein/nie + Komparativ

f) immer/zunehmend + Komparativ oder Komparativ + und + Komparativ

Unter den kontextbezogenen Kriterien sind folgende zu nennen:

a) direkte bzw. indirekte Nennung des zweiten Vergleichselements im vorhergehenden Text

b) Annahme eines bestimmten Vorwissens beim Adressaten (Präsupposition), eventuell wird dies durch einen Indikator wie wieder indiziert (z.B.: Ich bin jetzt wieder zufriedener in meinem Job).

c) offensichtliche Bezugnahme auf in der geschilderten Situation situativ gegebene außersprachliche Objekte. Die Bezugnahme wird häufig durch deiktische Ausdrücke wie hier, da, dieser usw. angezeigt (z.B.: Den finde ich schöner).

Bevor die Resultate der empirischen Untersuchung im Einzelnen dargestellt werden, sind noch die absoluten Komparativformen aufzulisten, auf die hin unser Korpus untersucht werden soll. Lange Zeit wurde von etlichen Autoren die Auffassung vertreten, der absolute Komparativ komme nur bei einer kleinen Gruppe von Adjektiven vor. So bemerkt beispielsweise Engel (1988:568), der absolute Komparativ sei auf die Adjektive alt, gut, herzlich, innig, jung, schön und wenige andere beschränkt. Diese Bemerkung ist in der Auflage 2004 von Engels Grammatik (344-346) nicht mehr zu finden. Nach Albers (2007:100) besteht keine Beschränkung für die absolute Komparation von Adjektiven. Sie kann den Gebrauch für mehr als nur eine kleine Gruppe von Adjektiven nachweisen, die sich zudem keinem bestimmten Adjektivtyp zuordnen lassen. Dieser Befund findet sich auch bei Thurmair (2001:228). Schefer-Krayenbühl (1989:76) setzt in ihrer korpusbasierten Untersuchung ebenfalls keine Beschränkung voraus. Die Liste der in ihrem Korpus am häufigsten auftretenden absoluten Komparative umfasst folgende Formen: höher, länger, älter, jünger, größer, kleiner, besser.

Die Komparativformen der Liste von Schefer-Krayenbühl sind auch in unserem Korpus bis auf eine noch zu ergänzende Form am häufigsten. Es sei darauf hingewiesen, dass das von Schefer-Krayenbühl zusammengestellte Korpus (1989:160) ebenfalls aus literarischen Texten besteht. ${ }^{11}$ Unser Korpus wurde deshalb auf die obengenannten Formen hin untersucht. Schefer-Krayenbühls Auswahl wurde durch die Komparativform öfter(s) ergänzt, welche am häufigsten in

11 Ihr Korpus besteht aus Werken folgender Autoren: Heinrich Böll, Lion Feuchtwanger, Thomas Mann, Albert Schweizer. 
unserem Korpus vorkommt. Neben öfter(s) treten von den von Schefer-Krayenbühl angegebenen Formen folgende mit einer besonders hohen Frequenz auf: älter, länger. Für die übrigen Formen größer, höher, jünger, kleiner, besser sind die Belege weniger häufig. Die Ergebnisse, die im Folgenden präsentiert werden, beziehen sich hier aus Platzgründen nur auf die Formen, die mit einer besonders hohen Häufigkeit vorkommen, d.h. öfter(s), älter, länger.

\section{Ergebnisse der empirischen Untersuchung}

Im Folgenden soll mittels des Vergleichs von Originaltextbeispielen und ihren Übersetzungen ins Italienische untersucht werden, welche Übersetzungsmöglichkeiten im Italienischen eingesetzt werden, um den deutschen absoluten Komparativ wiederzugeben.

Die Komparativform, die unter den zu untersuchenden Formen am häufigsten vorkommt, ist, wie oben gesagt, öfter(s). Die Darstellung der Ergebnisse beginnt mit dieser Form.

\section{1 öfter(s)}

Diese absolute Komparativform umfasst 126 Belege.

a) In $60 \%$ der Fälle (=76 Belege) wird öfter(s) mit dem Positiv wiedergegeben: spesso, sovente, di frequente/frequente (,oft, häufig'), molte volte (,vielmals'). Vgl.:

(5) Er sagte öfter solche Dinge (Zauberberg, 297) / Cose di questo genere ne diceva spesso (Montagna incantata, 376) / Diceva spesso cose di questo genere (Montagna magica, 332)

(6) Die Pferde zum Beispiel - ich hätte gehört, daß ihnen der Stier öfters den Bauch aufschlitze (Felix Krull, 400) / I cavalli per esempio - avevo sentito dire che sovente il toro fa loro uno squarcio nel ventre (Felix Krull, 341)

(7) „[...] Es ist besser, Sie geben die Sachen uns als ins Depot“, sagten sie, „denn im Depot kommen öfters Unterschleife vor [...]" (Prozeß, 9) / "É meglio che dia la roba a noi anziché in deposito" dissero, "perché in deposito le appropriazioni indebite sono frequenti [...]" (Processo, 1995, 17)

(8) in weißen Flanellhosen zum blauen Rock kam er öfters verspätet (Zauberberg, 667) / In calzoni di flanella bianca, che accompagnavano la giacca turchina, egli arrivava molte volte a tavola in ritardo (Montagna incantata, $889 \mathrm{f}$.) 
b) Durch die Übersetzung più volte (,mehrmals'), ripetutamente (,wiederholt‘) und parecchie volte (,mehrere Male') wird in $29 \%$ der Fälle (= 36 Belege) die durch öfter(s) charakterisierte Handlung auf der Eigenschaftsskala links von Handlungen und Vorgängen verortet, die oft stattfinden. Gleichzeitig liegt sie aber rechts vom Normalwert, d.h. rechts von Handlungen und Vorgängen, die mit durchschnittlicher Frequenz erfolgen, sowie rechts von Handlungen und Vorgängen, die nur manchmal eintreten. Diese Übersetzungen kennzeichnen einigermaßen adäquat den Abschnitt auf der Eigenschaftsskala, den der absolute Komparativ von oft abdeckt (s. u.a. Albers 2007:96, Abb. 3.12). Vgl.:

(9) er hatte es öfters aus den Gesprächen herausgehört (Zauberberg, 269) / più volte lo aveva dedotto ascoltando gli altri che conversavano (Montagna magica, 298)

(10) Öfters schon hatte er überlegt, ob es nicht gut wäre, eine Verteidigungsschrift auszuarbeiten und bei Gericht einzureichen (Prozeß, 98) / Già più volte aveva riflettuto se non fosse opportuno stendere una difesa e presentarla al tribunale. (Processo, 1975, 79) / Parecchie volte si era chiesto se non fosse bene stendere un memoriale e presentarlo al tribunale (Processo, 1995, 105)

(11) öfters hatte er schon verwundert auf einen kleinen, zweifellos nicht geheizten Eisenofen in der Ecke hingesehen, die Schwüle im Zimmer war unerklärlich (Prozeß, 127) / già parecchie volte si era meravigliato di vedere una piccola stufa di ferro, senza dubbio spenta, in un angolo, il caldo soffocante nella stanza era inspiegabile (Processo, 1989, 124)

(12) Besonders der letztere überragte $\mathrm{K}$. bedeutend und klopfte ihm öfters auf die Schulter (Prozeß, 8) / Quest'ultimo in particolare era molto più alto di K. e gli batté ripetutamente sulla spalla (Processo, 1995, 17)

c) Mit talvolta (,manchmal'), altre volte, qualche volta, certe volte (einige Male), alcune volte (,ab und $\mathrm{zu}^{\circ}$ ) und non di rado (,nicht selten'), die in sieben Belegen auftreten (6\%), wird eine Frequenz bezeichnet, die nicht hoch ist - sie liegt links vom Normalwert -, die aber ein Mindestmaß nicht unterschreitet. Vgl.:

(13) Darum gelangen mir diese Gedankenexperimente öfters recht gut, wenn auch nicht immer (Felix Krull, 241) / Questi esperimenti del mio pensiero mi riuscivano talvolta benissimo, seppur non sempre (Felix Krull, 208)

(14) Sie sprachen öfters auf eine Viertelstunde bei Anton Karlowitsch Ferge vor (Zauberberg, 400) / Stettero anche altre volte a chiacchierare un quarto d'ora con Anton Karlovic Ferge (Montagna incantata, 519)

(15) es wird einem öfter ganz ungemütlich, weil man sich - sagen wir mal - kontrolliert fühlt (Zauberberg, 142) / qualche volta si sta a disagio perché ci si sente - diciamo - sotto controllo (Montagna incantata, 166) 
(16) selbst mir, der ich dort zu Hause bin, ist es öfters kraß vorgekommen, wie ich nachträglich merke (Zauberberg, 261) / persino a me, che pure vivo in quell'ambiente, è sembrato certe volte inaudito, come mi accorgo ora (Montagna incantata, 327)

(17) Öfters drehte er sich vorsichtig um, ob der Polizeimann nicht folge (Prozeß, 192) / Si guardò indietro alcune volte cautamente per vedere se il poliziotto stava per aprir bocca (Processo, 1975, 156)

(18) auf der Brust nicht ganz festes Geschöpf, welches der Sänger jedoch, sei es um irgendwelcher Verfehlungen willen oder auch nur, um seiner allgemeinen Erbitterung Luft zu machen, öfters vermittels seiner Hosenträger empfindlich züchtigte (Felix Krull, 82) / una miseranda creatura forse debole di polmoni, che tuttavia il cantante non di rado, sia per le sue piccole colpe, sia per sfogare la propria amarezza, puniva energicamente a colpi di bretelle (Felix Krull, 77)

d) Zweimal (= $1 \%$ ) wird die Handlung bzw. der Vorgang als immer wieder auftretend aufgefasst und öfter(s) wird durch di continuo (,wiederkehrend') bzw. ogni volta (,jedes Mal') wiedergegeben, vgl.:

(19) so will ich ihr mütterliche Gefühle gar nicht bestreiten, aber eher als in lieben Blicken und Worten äußerten sie sich in kritischer Überwachung von Eleanors Tun und Lassen, zu der Mrs. Twentyman öfters die Schildplattlorgnette an die Augen führte (Felix Krull, 224) / Quanto a Mrs. Twentyman, non voglio negarle sentimenti materni, ma questi si manifestavano non tanto in sguardi o parole affettuose, bensì nella critica vigilanza di tutto quanto Eleanor faceva, un controllo per cui la signora portava di continuo l'occhialetto di tartaruga agli occhi (Felix Krull, 194)

(20) „Tu' ich öfters, wenn er so aufmuckt, und denke ja auch, er wird Räson annehmen [...]“ (Zauberberg, 448) / "Lo farò ogni volta che insorge in questo modo e penso che alla fine diventerà ragionevole [...]” (Montagna magica, 517)

e) Fünfmal (= $4 \%$ ) wird der absolute Komparativ nicht übersetzt. Vgl.:

(21) vor oder hinter [...] und dem Dermoplastiker, der sich aber öfter zu mir gesellte, um mir Erklärungen über die Landschaft, die Wunder der Pflanzenwelt zu geben (Felix Krull, 341) / col dermoplastico, o che questo si unisse a me per darmi spiegazioni circa il paesaggio e le meraviglie del mondo vegetale (Felix Krull, 293)

Im Folgenden werden die Ergebnisse zur Wiedergabe von öfter(s) tabellarisch dargestellt:

Positiv mehrmals usw. manchmal, ab wiederkehrend unübersetzt und zu usw.

\begin{tabular}{llllll}
\hline offer(s) & $60 \%$ & $29 \%$ & $6 \%$ & $1 \%$ & $4 \%$ \\
\hline
\end{tabular}




\section{2 länger}

Die Zahl der Belege betragen für länger im Korpus 107. Davon entfallen zwei auf ein Adverb (Positiv: lange) und 78 auf ein Adjektiv (Positiv: lang) in den festen Wendungen längere Zeit, eine längere Weile, seit längerem und des Längeren. Die übrigen 27 Belege entfallen auf ein Adjektiv in freien Kombinationen (z.B. ein längeres Warten).

Wir wenden uns zuerst den festen Wendungen zu. Hier wird auch die Adverbform länger behandelt, da diese mit den festen Wendungen sinnverwandt ist.

a) Am häufigsten (44\% = 35 Belege) werden die oben genannten Wendungen und das Adverb länger durch parecchio tempo (,ziemlich viel Zeit‘), un bel po', un bel pezzo (,ganz schön lange') und un periodo considerevole (,eine beträchtliche Zeit') übersetzt, wobei die Option parecchio tempo mit 28 Belegen eindeutig überwiegt. Diese Übersetzungen, die dem deutschen ziemlich lang(e) entsprechen, decken den Skalenbereich ab, der zwischen länger und dem Positiv lang(e) liegt (vgl. Abb. 1: Eine ziemlich lange Reise ist länger als eine längere Reise), und geben deshalb nur approximativ den Gradzustand des absoluten Komparativs wieder, vgl.:

(22) Sie, die nicht mehr las, seitdem sie nur noch strickte, mochte wohl wie Mama, die wegen Jan Bronski nicht mehr zum Lesen kam, die stattlichen Bände der Buchgemeinschaft, deren Mitglieder beide längere Zeit waren, an Leute verschenkt haben, die noch lasen (Blechtrommel, 100) / Così, come la mamma, che a causa di Jan Bronski non trovava più tempo per la lettura, avrà regalato i bei volumi del Club del Libro, di cui ambedue erano state per parecchio tempo socie, a persone che leggevano ancora (Tamburo di latta, 83)

(23) wo [in der Halle] man die Ärzte, die Oberin und anderes Personal noch längere Zeit an dem Sinnlosen hantieren sah, der dann auf eine Bahre davongetragen wurde (Zauberberg, 386) / dove ancora per un bel pezzo si videro trafficare i medici, la superiora e altro personale intorno a quell'uomo esanime che fu poi portato via su una barella (Montagna magica, 442)

(24) Wir werden sehen, wir sind noch auf längere Erdenzeit an diesem Lustort gebunden (Zauberberg, 458) / Staremo a vedere, saremo infatti legati a questo luogo di piacere ancora per un periodo considerevole del nostro tempo terreno (Montagna magica, 529)

(25) und dort setzte dann das Gespräch sich wohl noch eine längere Weile fort, der Verzögerung nach zu urteilen, womit der Assistent auf dem inneren Wege bei Joachim eintraf (Zauberberg, 467) / lì la conversazione pareva proseguire ancora per un bel po', almeno a giudicare dal ritardo con cui l'assistente arrivava da lui, Joachim, passando per la via interna (Montagna magica, 541) 
b) In $25 \%$ der Fälle (=20 Belege) greifen die Übersetzer zu Wiedergaben wie certo tempo (,eine gewisse Zeit'), qualche tempo, un po', un pezzo, da tempo (,eine Weile, etwas Zeit'). Mit diesen Ausdrücken wollen oder können die Übersetzer die angegebene Länge nicht näher bezeichnen, da sie sie auf einen ziemlich breiten Skalenbereich festlegen, der sich vom Pol kurz bis zum Normalwert erstreckt. Diese Übersetzungen decken den Geltungsbereich des absoluten Komparativs nicht ab. Vgl.:

(26) Acht Betten mit grauen Friesdecken und flachen, sichtlich längere Zeit nicht gewaschenen Kopfkissen waren kojenartig je zwei und zwei übereinander an den Seitenwänden angeordnet (Felix Krull, 143) / Lungo le pareti erano disposte a modo di cuccette due file sovrapposte di quattro letti con grigie coperte e cuscini appiattiti, evidentemente non lavati da un pezzo (Felix Krull, 126)

(27) Wenn es immer etwas Beschämendes hat, sich längere Zeit allein und unbeobachtet geglaubt zu haben (Felix Krull, 146) / È sempre mortificante essersi creduti soli e inosservati per un certo tempo (Felix Krull, 128)

(28) K. sah sie mit zusammengezogenen Augen an. „Gewiß“, sagte er, „Sie wohnen doch schon längere Zeit bei Frau Grubach“ (Prozeß, 70) / K. stringendo le palpebre rispose: “Certo. Lei abita già da un po' qui dalla signora Grubach”. (Processo, 1975, 55)

(29) K., der schon lange keine Bilder gesehen hatte, betrachtete den Ritter längere Zeit, obwohl er immerfort mit den Augen zwinkern mußte (Prozeß, 175) / K., che da tempo non vedeva dei quadri, osservò a lungo il cavaliere, pur dovendo continuare a strizzare gli occhi (Processo, 1989, 166)

c) In $25 \%$ (= 20 Belege) wird der absolute Komparativ als Positiv aufgefasst und durch lungo tempo, molto tempo, tanto tempo (,lange Zeit'), a lungo, tanto (,lange'), un lungo periodo (,ein langer Zeitabschnitt') übersetzt, vgl.:

(30) Wir standen uns längere Zeit verlegen lächelnd gegenüber (Blechtrommel, 297) / Ci guardammo a lungo con un sorriso imbarazzato (Tamburo di latta, 247)

(31) Tatsächlich erwachte er längere Zeit, bevor es an seine Tür klopfte (Zauberberg, 783) / Di fatti fu sveglio molto tempo prima che bussassero alla sua porta (Montagna incantata, 1048)

(32) Froh erhob sich in mir, was ich je aus dem Munde meines Paten Schimmelpreester, der $\underline{\text { des }}$ öfteren und längeren in jenem sonnigen Lande gewesen war, von italienischen Lauten gehört hatte (Felix Krull, 162) / Sorse lietamente in me ogni suono italiano che mai avessi udito dalla bocca del mio padrino Schimmelpreester, che sovente e a lungo era stato in quel paese del sole (Felix Krull, 141). 
d) In drei Fällen (4 \%) bleibt der absolute Komparativ unübersetzt. Vgl.:

(33) Als er mich gestern besuchte, tat er erst längere Zeit geheimnisvoll (Blechtrommel, 79) / Aveva un fare misterioso ieri quando è venuto a trovarmi (Tamburo di latta, 65)

e) In einem Fall (= 1\%) wird die Komparativform durch eine Periphrase mit dem Verb continuare (,fortsetzen') wiedergegeben. Vgl.:

(34) Längere Zeit mütterliches und väterliches Versprechen gegeneinander abwägend, beobachtete und belauschte ich, Oskar, einen Nachtfalter, der sich ins Zimmer verflogen hatte (Blechtrommel, 47) / Mentre continuavo a soppesare e a comparare i propositi paterni e materni io, Oskar, mi misi a osservare e ad ascoltare una falena smarrita nella camera. (Tamburo di latta, 38)

f) In einem Fall wird der absolute Komparativ durch den relativen übersetzt. Vgl.:

(35) Des längeren noch, und wiederholt, hatten sie mit dem Literaten über seinen Entschluß gesprochen (Zauberberg, 456) / Più a lungo poi e ripetute volte gli avevano parlato di quella sua decisione (Montagna incantata, 596).

Im Folgenden werden die Ergebnisse zu länger, soweit es als Adjektiv in festen Wendungen vorkommt, sowie zum Adverb länger zusammengefasst:

\begin{tabular}{rrrrrrr}
\hline & Positiv & $\begin{array}{r}\text { ziemlich } \\
\text { lange }\end{array}$ & $\begin{array}{r}\text { ungenaue } \\
\text { Bezeichnung }\end{array}$ & $\begin{array}{r}\text { Periphrase } \\
\text { mit fortsetzen }\end{array}$ & rel. Komp. unübersetzt \\
\hline länger & $25 \%$ & $44 \%$ & $25 \%$ & $1 \%$ & $1 \%$ & $4 \%$ \\
\hline
\end{tabular}

Anders verhält es sich, wenn länger als Adjektiv in freien Kombinationen vorkommt (27 Belege).

g) Hier überwiegt eindeutig die Übersetzung durch den Positiv (78 \%). Die Übersetzungen mit Positiv sind lungo, prolungato (,lang') und tanto tempo (,lange Zeit'). Vgl.:

(36) Längeres Warten wirkt sich erzieherisch aus. Es kann aber auch längeres Warten den Wartenden dazu verführen, die zu erwartende Begrüßungsszene so ins Detail gehend auszumalen, daß dem Erwarteten jede Chance einer geglückten Überraschung genommen wird. (Blechtrommel, 252) / Una lunga attesa ha un effetto educativo. Ma un'attesa prolungata può anche indurre chi attende a raffigurarsi la scena attesa dell'incontro con tanti particolari che all'atteso viene tolta ogni possibilità di coglierlo di sorpresa. (Tamburo di latta, 209)

(37) Ehrlich erfreut vielmehr, ihm nach längerer Trennung wieder zu begegnen (Zauberberg, 473) / Sinceramente lieti, invece, d'incontrarlo dopo tanto tempo (Montagna incantata, 620) 
h) In $11 \%$ der Fälle wird der Positiv durch piuttosto leicht abgeschwächt. Diese Formulierung drückt aus, dass die bezeichnete Eigenschaft in ausreichendem Maße vorhanden ist, dass aber der durch den Positiv bezeichnete Punkt auf der Skala nicht völlig erreicht wird. Diese Übersetzung referiert auf einen Geltungsbereich, der zwischen dem Normalwert und dem durch ziemlich lang bezeichneten Abschnitt zu verorten ist. Sie deckt den Geltungsbereich des absoluten Komparativs ab. Vgl.

(38) Ein längerer Tisch schnitt schräg zur Rechten einen Winkel des Zimmers ab (Felix Krull, 101) / Una tavola piuttosto lunga tagliava a destra un angolo del locale (Felix Krull, 92)

i) Durch die Übersetzung da tempo (,seit einiger Zeit‘) verzichtet der Übersetzer auf eine nähere Bestimmung der bezeichneten Länge (11 \%). Der damit abgedeckte Skalenbereich ist hier wie in 5.2.b ziemlich breit und zwischen dem Normalwert und dem Pol kurz zu verorten. Vgl.:

(39) weil er die Miene hatte, als ob er von längerer Hand her sich auf die Sache verstände (Zauberberg, 804) / perché lui aveva l'aria di uno che da tempo si intendesse di simili faccende (Montagna magica, 952).

Hier eine tabellarische Übersicht über die Ergebnisse zu länger, soweit es als Adjektiv in freien Kombinationen vorkommt:

\begin{tabular}{lrrr}
\hline & Positiv & ungenaue Bezeichnung & piuttosto + Adj. \\
\hline länger & $78 \%$ & $11 \%$ & $11 \%$ \\
\hline
\end{tabular}

\section{3 älter}

Zuerst werden die Belege analysiert, in denen das Adjektiv in Verbindung mit einer Person vorkommt (ältere Frau, älterer Herr usw.). Die Belege dafür sind insgesamt 49.

a) In $61 \%$ der Fälle (= 30 Belege) wird die absolute Komparativform durch anziano (im vorgerückten Alter, aber auch euphemistisch für alt) wiedergegeben. Ob diese Übersetzung als angemessen $\mathrm{zu}$ betrachten ist, wird in Abschnitt 6 erörtert. Vgl.:

(40) Eine Frau ging im Garten umher, eine ältere Dame von düsterem, ja tragischem Aussehen (Zauberberg, 65) / Una donna camminava su e giù per il giardino, era una signora anziana dall'aspetto tetro, addirittura tragico (Montagna magica, 56) 
(41) Diese Plaketten wurden zumeist von älteren Herren und gebrechlichen Frauen [...] an den Mantelaufschlägen getragen (Katz und Maus, 54) / Queste plachette venivano in genere adoprate da signori anziani e da fragili signore (Gatto e topo, 66)

b) Denselben Eigenschaftsgrad wie anziano (in der Bedeutung ,im vorgerückten Alter') bezeichnet auf der Eigenschaftsskala das Adjektiv attempato und die Periphrase in età avanzata (,im vorgerückten Alter'). Für diese Übersetzungsvarianten sind nur zwei Belege zu finden (= 4 \%). Vgl.:

(42) Eine Frau ging im Garten umher, eine ältere Dame von düsterem, ja tragischem Aussehen (Zauberberg, 65) / Nel giardino passeggiava una donna attempata di aspetto tetro, persino tragico (Montagna incantata, 63)

(43) Ein ehemaliger Bildhauer aus der österreichischen Provinz, ein schon älterer Mann mit weißem Schnurrbart, einer Hackennase und blauen Augen, hatte einen Plan finanzpolitischer Art gefaßt (Zauberberg, 795) / Un ex scultore, venuto dalle provincie austriache, persona già in età avanzata coi baffi bianchi, il naso aquilino e gli occhi azzurri, aveva concepito un piano di politica finanziaria (Montagna incantata, 1064)

c) In $13 \%$ der Fälle (= sechs Belege) wird die Komparativform durch den Positiv vecchio (,alt') wiedergegeben. Vgl.:

(44) eine ältere Hebamme, die nur noch dann und wann zu ihrem Köfferchen griff, mußte aus der nahen Hertastraße gerufen werden (Blechtrommel, 46f.) / Si dovette chiamare dalla vicina Hertastrasse una vecchia levatrice che solo di quando in quando ritirava fuori la sua valigetta (Tamburo di latta, 35)

d) Für maturo (,reif') und di età matura (,in reifem Alter') finden sich zwei Belege (= 4 \%). Diese Übersetzungen sind auf einem Abschnitt der Eigenschaftsskala zu verorten, der annähernd dem Normalwert entspricht. Sie geben deshalb die Bedeutung des absoluten Komparativs nicht adäquat wieder. Vgl.:

(45) Mynheer Peeperkorn, ein älterer Holländer, war eine Zeitlang Gast des Hauses „Berghof“" (Zauberberg, 689) / Mynheer Peeperkorn, un olandese di età matura, fu per qualche tempo ospite del Berghof (Montagna incantata, 919)

e) Die Periphrase di una certa età (,von einem gewissen Alter'), die nur sehr indirekt auf das tatsächliche Alter verweist, kommt nur einmal vor (= $2 \%)$ (vgl. 5.2.b und 5.2.i). Vgl.:

(46) Die Muse Ulla aber, [...] erwärmte sich derart für die neuen Tapeten, daß sie den Maler Lankes, der sie verlassen hatte, schnell vergaß und die verschieden großen Dekorationen eines schon älteren Malers, Meitel mit Namen, hübsch, lustig, drollig, phantastisch, enorm und sogar chic fand (Blechtrommel, 586) / La musa Ulla invece, [...] si entusiasmò talmente per le nuove tappezzerie che dimenticò presto il pittore Lankes, dal quale era stata abbando- 
nata, e trovò graziose, buffe, divertenti, fantastiche, enormi e persino chic le composizioni decorative di diverse misure eseguite da un pittore di una certa età, di nome Meitel (Tamburo di latta, 487)

f) Das Adverb piuttosto modifiziert mit abschwächender Wirkung fünfmal das Adjektiv anziano (= $11 \%$ ) und einmal attempato (2\%) (s. auch 5.2.h). Inwieweit diese Übersetzungen angemessen bzw. unangemessen sind, wird in 6 . erörtert. Vgl.:

(47) Im Licht der Kerze, die der Onkel jetzt hochhielt, sah man dort, bei einem kleinen Tischchen, einen älteren Herrn sitzen (Prozeß, 91) / Alla luce della candela che ora lo zio teneva sollevata vi si vide un uomo piuttosto anziano seduto a un tavolinetto (Processo, 1975, 73)

g) Zweimal (= $3 \%$ ) wird der absolute Komparativ durch den relativen wiedergegeben. Vgl.:

(48) In diesem Frühjahr pflegte K. die Abende in der Weise zu verbringen, daß er nach der Arbeit [...], einen kleinen Spaziergang allein oder mit Beamten machte und dann in eine Bierstube ging, wo er an einem Stammtisch mit meist älteren Herren gewöhnlich bis elf Uhr beisammensaß. (Prozeß, 20) / In quella primavera K. aveva l'abitudine, dopo il lavoro [...] di fare una piccola passeggiata da solo o con gli impiegati, poi andava in una birreria dove si intratteneva a un tavolo riservato, di solito fino alle undici, insieme con persone quasi sempre più anziane di lui (Processo, 1989, 30)

Die Resultate zu älter, soweit es in Verbindung mit einer Personenbezeichnung auftritt, können folgendermaßen zusammengefasst werden:

\begin{tabular}{rrrrrrrr}
\hline & Positiv & anziano & $\begin{array}{r}\text { ungenaue } \\
\text { Bezeichnung }\end{array}$ & $\begin{array}{r}\text { reif } \\
\text { rückten Alter }\end{array}$ & $\begin{array}{r}\text { im vorge- } \\
\text { piuttosto + Adj. }\end{array}$ & rel. Komp. \\
\hline älter & $13 \%$ & $61 \%$ & $2 \%$ & $4 \%$ & $4 \%$ & $13 \%$ & $3 \%$ \\
\hline
\end{tabular}

Älter in Verbindung mit einer Sachbezeichnung wird regelmäßig mit dem Positiv vecchio übersetzt. Es gibt insgesamt fünf Belege. Vgl.:

(49) während er zusah, wie Joachim sich zur Liegekur fertig machte, seine litewkaartige Hausjoppe anlegte, darüber einen älteren Paletot zog (Zauberberg, 125) / mentre guardava Joachim che si preparava alla cura, indossava la giubba da casa simile a una casacca militare, s'infilava sopra a questa un vecchio cappotto (Montagna incantata, 145) / mentre osservava Joachim che si preparava per la cura del riposo, indossava la giacca da camera e una litewka su cui infilava un vecchio paltò (Montagna magica, 127) 


\section{Auswertung der Ergebnisse}

Im Folgenden werden die eben präsentierten Übersetzungsmöglichkeiten und die entsprechenden Prozentsätze im Einzelnen erläutert.

öfter(s): Die überwiegende Mehrheit der Übersetzungen wählt die Positivform aus (60\%). Dies ist auffallend, zumal öfter(s) zu den absoluten Komparativformen gehört, die sowohl in DaF-Wörterbüchern als auch in einsprachigen sowie in zweisprachigen Wörterbüchern lexikalisiert ist. Obwohl die Form öfters in Wörterbüchern als eigenes Lemma geführt ist, wird sie nicht als Belegbeispiel von den herangezogenen Fremdsprachengrammatiken für Italiener und nicht von den führenden deutschen Grammatiken (Duden, Engel, Helbig-Buscha) angeführt. ${ }^{12}$ Die Prozentsätze der Übersetzungen, die den absoluten Komparativ treffend (29 \%) bzw. nur annährend treffend (6\%) wiedergeben, sind deutlich geringer als der Prozentsatz, der die Wiedergabe durch den Positiv angibt. Den gesammelten Daten ist ferner zu entnehmen, dass öfters neben länger am häufigsten (4\%) nicht übersetzt wird. Eine adäquate Wiedergabe im Italienischen ist nur durch eine Periphrase möglich (s. 5.1.b: parecchie volte, più volte).

länger: Bei der Wiedergabe von länger fällt auf, dass die unzutreffende Übersetzung durch den Positiv bei freien Kombinationen beträchtlich höher ist (78 \%) als bei festen Wendungen ( $25 \%$ ). Die Identifizierung des absoluten Komparativs in Kombinationen und Verbindungen, die nicht feststehend sind, fällt den Übersetzern deutlich schwerer als bei feststehenden Kombinationen. Die hier infrage kommenden festen Wendungen (längere Zeit, längere Weile, seit Längerem, des Längeren) sowie das Adverb länger sind zu den Komparativformen zu zählen, die ziemlich häufig sowohl in den Fremdsprachengrammatiken für Italiener als auch in den gängigen deutschen Grammatiken als Beispiele angeführt werden. Die Übersetzer scheinen mit diesen Formen vertrauter zu sein als mit dem in den Grammatiken kaum zitierten öfter(s). Unter den Übersetzungsvarianten, die den absoluten Komparativ mit einer gewissen Annährung wiedergeben, ist die durch parecchio tempo (,ziemlich viel Zeit'), un bel po', un bel pezzo (,ganz schön lange‘) und un periodo considerevole (,beträchtliche Zeit') am häufigsten (5.2.a: 44 \%). So hoch wie der Prozentsatz der Wiedergaben, die auf eine genaue Bezeichnung der Länge verzichten, ist der Prozentsatz der Übersetzungen durch den Positiv (25\%). Irreführende Übersetzungen durch den Positiv, wie z. B. a lungo, per lungo tempo, finden sich auch in zweisprachigen Wörterbüchern (s. Sansoni ab 1996).

12 Die von Schefer-Krayenbühl in ihrer Untersuchung erstellte Liste enthält diese Form auch nicht. Obwohl ihr Korpus ebenfalls aus literarischen Texten besteht, scheint diese Form in ihrem Korpus nicht so häufig aufzutreten wie in unserem. 
Die Form länger wird ansonsten in einsprachigen Wörterbüchern nicht immer als lexikalisiert erfasst und nur selten in DaF-Wörterbüchern (s. S. 89f.). Auch bei länger ist eine angemessene Wiedergabe im Italienischen nur durch eine Periphrase möglich.

älter: Bei der Wiedergabe dieser absoluten Komparativform in Verbindung mit einer Person wird eine breitere Palette an Übersetzungsmöglichkeiten angeboten als bei den anderen untersuchten Formen. Diese Form ist die meist diskutierte in der einschlägigen Literatur, vor allem was ihr Vorkommen in der Phrase eine ältere Dame bzw. ein älterer Herr betrifft. Diesbezüglich macht die Literatur immer wieder auf die beschönigende Verwendung von älter aufmerksam (s. z. B. Becker 2005:110, Trost 2006:85). In dieser Verwendungsweise bezieht sich älter auf eine Person, die sich nicht im Sinne des absoluten Komparativs dem Alt-Sein annähert, sondern im Sinne des Positivs bereits alt ist. Mit diesem Komparativ wird somit keine modifizierende Abschwächung des Positivs ausgedrückt, er hat vielmehr eine auf Höflichkeit oder Vorsicht basierende Abmilderungsfunktion. Die semantische Identität mit dem Positiv alt bleibt bewahrt, es kommt lediglich die pragmatische Funktion der Milderung zum Ausdruck.

In der Mehrheit der Fälle entscheiden sich die Übersetzer zur Wiedergabe von älter in Verbindung mit einer Person für das Adjektiv anziano (61 \%). Das Adjektiv anziano bedeutet ursprünglich, jemand, der im fortgeschrittenen, aber noch nicht im hohen Lebensalter ist' (Zanichelli 2010) und ist mit vecchio (,im hohen Alter, alt') nicht gleichbedeutend. In dieser Lesart entspricht anziano dem deutschen absoluten Komparativ älter mit abschwächender Funktion. Im Laufe der Zeit hat aber anziano eine semantische Veränderung erfahren und gilt heutzutage vornehmlich als euphemistische Form für den Positiv vecchio (,alt'), der negativ besetzt ist und von dessen Gebrauch in Bezug auf Personen abgeraten wird (s. Beccaria 2006:48, Bencini/Manetti 2005:227). In dieser zweiten, vorherrschenden Lesart entspricht anziano gut dem absoluten Komparativ älter, wenn dieser aus Gründen der Vorsicht oder der Höflichkeit verwendet wird. Inwieweit ist anziano eine angemessene Übersetzung für älter, falls die höfliche Lesart nicht infrage kommt und der absoluten Komparativform nur eine abschwächende Funktion beizumessen ist? Da anziano derzeit primär als beschönigende und mildernde Umschreibung für alt aufzufassen ist, ist es in einem solchen Fall als unadäquate Übersetzung einzuschätzen. Hier sind Übersetzungsvarianten wie di età avanzata, piuttosto anziano, piuttosto attempato (s. 5.3.b; 5.3.f) angemessener, weil sie die abschwächende Komponente der Komparativform gegenüber dem Positiv eindeutig ausdrücken, was durch die Übersetzung anziano eben nicht gesichert ist. Die Übersetzer müssen je nach Kontext bestimmen, ob die höfliche oder die abschwächende Lesart vorwiegt. Bei Beleg (42) ist z. B. die abschwächende Lesart 
die einzige, die angemessen ist. Deshalb wäre einer der oben genannten Übersetzungsvorschläge zutreffender als die verwendete Form anziana.

Besondere Aufmerksamkeit ist der Übersetzung von älter + Personenbezeichnung auch deshalb zu widmen, da im selben Text die Kombination älter + Personenbezeichnung und die Verbindung alt + Personenbezeichnung vorkommen können (z. B. ältere Frau neben alte Frau). Falls beide Formen gleichzeitig im selben Text vorhanden sind, sollten die absolute Komparativform älter und der Positiv alt nicht identisch mit anziano übersetzt werden (z. B. anziana signora). Wenn sich der Übersetzer bzw. die Übersetzerin zur Wiedergabe sowohl des Positivs als auch des absoluten Komparativs für dieselbe Form entscheiden, sind die Positivform und der absolute Komparativ für das italienische Lesepublikum nicht mehr zu unterscheiden. ${ }^{13}$

In $13 \%$ der Fälle wird der absolute Komparativ in Verbindung mit einer Person nicht als solcher erkannt und durch den negativ besetzten Positiv vecchio (,alt ${ }^{\star}$ ) wiedergegeben. Warum werden diese Belege nicht mit anziano, sondern mit vecchio übersetzt, dessen Verwendung in Bezug auf Personen heutzutage nicht empfohlen wird? Wenn man die Belege näher betrachtet, so ist festzustellen, dass alle sechs Belege in Übersetzungen vorkommen, die aus den 70er Jahren stammen, als die Lesart von anziano als Euphemismus für alt noch nicht vorherrschend war. Sie treten außerdem größtenteils in Verbindung mit der Pluralform ältere Herren auf. ${ }^{14}$ Bei solch einer unbestimmten Pluralform scheint es den Übersetzern nicht erforderlich zu sein, auf eine höfliche Umschreibung für das Alter zurückzugreifen.

Für die Verbindung von älter mit einer Sache gibt es nur fünf Belege. Bei diesen Belegen wird kein Versuch unternommen, den absoluten Komparativ wiederzugeben. Bei Beleg (49) wird z.B. ein möglicher Übersetzungsvorschlag wie piuttosto vecchio, der adäquat den absoluten Komparativ übersetzen könnte (s. 5.2.h), nicht eingesetzt. Das kann z.T. darauf zurückgeführt werden, dass die Komparativform älter sowohl in Wörterbüchern als auch in Grammatiken nur in Kombination mit einer Personenbezeichnung als Beispiel angeführt wird. In nicht

13 In La montagna magica (2010) werden z. B. sowohl eine alte Dame als auch eine ältere Dame größtenteils durch una signora anziana wiedergegeben; anders verhält es sich in La montagna incantata (1965), wo der Übersetzer die beiden Formen durch unterschiedliche Übersetzungen getrennt hält. Der Positiv wird nämlich durch una vecchia signora wiedergegeben, der absolute Komparativ durch una anziana signora. Vecchio + Personenbezeichnung scheint in den 70er Jahren noch nicht negativ besetzt zu sein, im Gegensatz zum heutigen Sprachgebrauch.

14 Vgl. u.a.: es mußten die älteren Herren [...] vorsichtig sein (Blechtrommel, 633) / $\underline{\mathrm{i} \text { vecchi }}$ signori [...] dovevano stare attenti (Tamburo di latta, 525); Sie hielt einen schwarzen Regenschirm, wie ihn ältere Herren bei sich führen, gegen die Sonne (Blechtrommel, 633) / Teneva contro il sole un ombrello nero come quello dei vecchi signori (Tamburo di latta, 546). 
vertrauten Kontexten wird daher die absolute Komparativform nicht wahrgenommen.

\section{Fazit}

Die Ergebnisse der Korpusanalyse zeigen, dass das Phänomen des absoluten Komparativs den Übersetzern nicht sehr geläufig ist. Das wird klar, wenn man sich die Prozentsätze der Wiedergabe durch den Positiv vergegenwärtigt. Diese Prozentsätze sind relativ hoch: Sie schwanken zwischen $25 \%$ (s. 5.2.c bei dem Adverb lange) und $78 \%$ (s. 5.2.g bei dem Adjektiv lang). Bei der Form älter in Verbindung mit einer Personenbezeichnung ist der Prozentsatz der Wiedergabe durch den Positiv alt (,vecchio') (5.3.c: 13 \%) nur scheinbar niedriger, da die sehr oft auftretende Form anziano (61\%), wie oben ausgeführt, vornehmlich als euphemistische Umschreibung für den Positiv alt aufzufassen ist. Die aus der Analyse gewonnenen Resultate bestätigen, was in dieser Untersuchung bezüglich der Behandlung des absoluten Komparativs in den für das italienische Publikum geschriebenen Fremdsprachengrammatiken bereits festgestellt wurde: Dem Phänomen wird nicht genügend Beachtung geschenkt. Insbesondere wird nicht hinreichend beachtet, welche spezifische Bedeutung der absolute Komparativ im Vergleich zum Positiv hat. Die durchgeführte Analyse hat außerdem gezeigt, dass die Formen, die am wenigsten in den Fremdsprachengrammatiken als Belege angeführt werden (öfter(s), länger in freien Verbindungen), am häufigsten unzutreffend mit dem Positiv übersetzt werden, obwohl gerade diese Formen in der Regel in der Lexikographie als feststehende Ausdrücke behandelt werden.

Sofern die absoluten Komparativformen nicht durch den reinen Positiv wiedergegeben werden, gehen die Übersetzungsvarianten, die den Positiv zu modifizieren versuchen, nach Ausweis unserer Daten hauptsächlich in drei Richtungen, wobei zu merken ist, dass die einzelnen Übersetzer bei ihren Übersetzungen konsequent bleiben. Die drei Richtungen können wie folgt zusammengefasst werden:

1) Die Übersetzer verzichten auf eine nähere Bestimmung der betreffenden Eigenschaft, indem sie Ausdrücke wie certo (,gewiss'), qualche (,einige'), $d a$ tempo (,seit einiger Zeit') verwenden. Diese Ausdrücke benennen eine nicht starke Ausprägung der Eigenschaft, die aber in einem Mindestmaß vorhanden ist. Solche Formulierungen referieren auf einen ziemlich breiten Skalenbereich, der aber nicht dem des absoluten Komparativs entspricht (s. 5.2.b, 5.2.i, 5.3.e).

2) Der Positiv wird durch das Adverb piuttosto modifiziert, das eine abschwächende Funktion erfüllt. Diese Übersetzungsvariante wird seltener eingesetzt 
(s. 5.2.h, 5.3.f) als die erste, obwohl doch gerade sie einigermaßen adäquat auf den Geltungsbereich des absoluten Komparativs referiert.

3) Der Positiv wird durch Elemente (parecchio, un bel...) modifiziert, die einen Grad auf der Eigenschaftsskala belegen, der der deutschen Umschreibung ziemlich + Adjektiv entspricht. Somit wird ein Eigenschaftsgrad bezeichnet, der auf der Skala zwischen der Bedeutung der absoluten Komparativform und des Positivs steht (s. 5.2.a: Eine längere Zeit ist kürzer als eine ziemlich lange Zeit).

Das dargestellte Repertoire an Ausdrücken, das im Italienischen zur Wiedergabe des absoluten Komparativs zur Verfügung steht, stimmt im Großen und Ganzen mit dem überein, das die in dieser Untersuchung herangezogenen Fremdsprachengrammatiken anbieten (s. S. 89). Eine besondere Aufmerksamkeit galt in unseren Ausführungen der Wiedergabe der Form älter durch anziano. Dazu wurde festgestellt, dass anziano heutzutage den absoluten Komparativ älter nur dann adäquat wiedergeben kann, wenn der Komparativ aus Gründen der Vorsicht und der Höflichkeit eingesetzt wird. Die untersuchten Grammatiken bieten zur Übersetzung der Form älter unterschiedliche Optionen an (sowohl anziano als auch piuttosto anziano, s. S. 87), ohne auf die beiden Lesarten von anziano aufmerksam zu machen.

Von den drei oben präsentierten Übersetzungsmöglichkeiten, zu denen die Übersetzer greifen, falls sie sich nicht für den Positiv entscheiden, ermöglicht nur die zweite Option eine Übersetzung des absoluten Komparativs, die annähernd seine abschwächende Funktion wiedergibt. Wenn man von der oben besprochenen Form anziano absieht, ist eine adäquate Übersetzung des absoluten Komparativs ins Italienische bei den hier behandelten Fällen (öfter(s), länger, älter) nur durch eine Periphrase möglich. Interessant wäre es sicherlich, die Wiedergabe der absoluten Komparativform größer anhand unseres Korpus zu untersuchen, da sie laut den herangezogenen Grammatiken auch durch ein Einzelwort (cospicuo, considerevole) wiedergegeben werden kann. Aus Platzgründen wurde dies hier nicht durchgeführt.

Zusammenfassend: Es wäre sicherlich nützlich, die Spezifizität des deutschen absoluten Komparativs in Fremdsprachengrammatiken und kontrastiven Studien Italienisch/Deutsch eingehender zu behandeln. Wenn sie Wesen und Gebrauch des absoluten Komparativs befriedigend würdigen könnten, würde dies sich sicherlich auch auf die Wiedergabe des Phänomens in Übersetzungstexten positiv auswirken. In den Vordergrund zu stellen wäre insbesondere die Semantik des absoluten Komparativs, d.h. seine abschwächende Funktion zum Positiv, die sowohl regressiv als auch progressiv auf der Bewertungsskala erfolgen kann (s. S. 81). Dieser Aspekt ist besonders hervorzuheben, zumal der absolute Kompara- 
tiv, wie oben angemerkt, viel weiter verbreitet ist, als lange angenommen wurde, und sich nicht auf eine bestimmte Anzahl von Adjektiven beschränkt. Bei den in der vorliegenden Analyse untersuchten absoluten Komparativen (öfters, länger, älter) handelt es sich um Formen, deren Positive (oft, lang(e), alt) auf der Bewertungsskala die Zielantonyme darstellen. Die abschwächende Funktion, die den analysierten absoluten Komparativformen im Vergleich zum Positiv zukommt, erfolgt deshalb relativ zum Normalwert progressiv. Die hier präsentierten Übersetzungen beziehen sich daher nur auf diese Art von Komparativformen. Aufschlussreich wäre es, anhand von einem Korpus die Wiedergabe von absoluten Komparativen wie seltener, kürzer, jünger ins Italienische zu untersuchen, deren Positive auf der Bewertungsskala die entsprechenden Ausgangsantonyme darstellen und deren abschwächende Funktion relativ zum Normalwert regressiv erfolgt. Es wäre u.a. zu ermitteln, ob der Prozentsatz der Übersetzungen durch den Positiv bei diesen Formen ebenso hoch ist, wie er in der vorliegenden Analyse ermittelt wurde, und welche Übersetzungsvorschläge angeboten werden.

\section{Primärliteratur}

Grass, Günther (1959): Die Blechtrommel. Darmstadt und Neuwied: Hermann Luchterhand Verlag. 1979.

ders. (1962): Il tamburo di latta. Übersetzung von Lia Secci. Milano: Feltrinelli. ders. (1974): Katz und Maus. Darmstadt: Luchterhand Verlag.

ders. (2009): Gatto e topo. Übersetzung von Enrico Filippini. Milano: Feltrinelli. Kafka, Franz (1925): Der Prozeß. Frankfurt am Main: Fischer Verlag. 1979. ders. (1975): Il processo. Übersetzung von Ervino Pocar. Milano: Arnoldo Mondadori. ders. (1983): Il processo. Übersetzung von Primo Levi. Torino: Giulio Einaudi.

ders. (1989): Il processo. Übersetzung von Giuseppe Landolfi Petrone und Maria Martorelli.

Milano: Newton Narrativa.

ders. (1995): Il processo. Übersetzung von Anita Raja. Milano: Feltrinelli.

Mann, Thomas (1912): Der Tod in Venedig. Frankfurt am Main: Fischer Verlag. 1979.

ders. (1970): La morte a Venezia. Übersetzung von Emilio Castellani. Milano: Arnoldo Mondadori. ders. (2004): La morte a Venezia. Übersetzung von Anita Rho. Torino: Einaudi Editore. ders (2010): La morte a Venezia. Übersetzung von Emilio Castellani, durchgesehen von Renata Colorni. Milano: Arnoldo Mondadori.

ders. (1924): Der Zauberberg. Frankfurt am Main: Fischer Verlag. 1991.

ders. (1965): La montagna incantata. Übersetzung von Ervino Pocar. Milano: Arnoldo Mondadori. ders. (2010): La montagna magica. Übersetzung von Renata Colorni. Milano: Arnoldo Mondadori. ders. (1954): Bekenntnisse des Hochstaplers Felix Krull. Frankfurt am Main: Fischer Verlag. 1985. ders. (1965): Confessioni del cavaliere d'industria Felix Krull. Übersetzung von Lavinia Mazzucchetti. Milano: Arnoldo Mondadori. 


\section{Sekundärliteratur}

Albers, Janine (2007): Der absolute Komparativ: eine empirische Untersuchung zu seiner Bedeutung und kommunikativen Verwendung. Göttingen: Cuvillier.

Beccaria, Gian Luigi (2006): Per difesa e per amore. La lingua italiana oggi. Milano: Garzanti.

Becker, Thomas (2005): „Warum eine ältere Dame älter ist als eine ältere Dame: Zum absoluten Komparativ im Deutschen." Deutsche Sprache 33, 97-116.

Blasco, Ferrer (1999): Italiano e tedesco: un confronto linguistico. Torino: Paravia Scriptorium.

Bencini, Andrea / Manetti, Beatrice (2005): Le parole dell'Italia che cambia. Grassina (Firenze): Le Monnier Università.

Cartagena, Nelson / Gauner, Hans-Martin (1989): Vergleichende Grammatik Spanisch-Deutsch. Duden-Sonderreihe vergleichende Grammatiken. 2 Bde. Mannheim et al.: Dudenverlag.

Catalani, Luigi (2004): Deutsch, Französisch und Spanisch im Kontrast mit dem Italienischen. Frankfurt am Main u. a.: Peter Lang.

Cinato Kather, Lucia (2011): Mediazione linguistica tedesco-italiano: aspetti teorici e applicativi. Milano: Hoepli.

Coletsos Bosco, Maria Sandra / Costa, Marcella (2004): Italiano e tedesco: un confronto. Alessandria: Ed. dell'Orso.

Dethloff, Uwe / Wagner, Horst (2007): Die französische Grammatik. 2. Auflage. Tübingen u. a.: Francke.

Eisenberg, Peter (2004): Grundriß der deutschen Grammatik. 2. Auflage. Stuttgart: Metzler. Engel, Ulrich (1988): Deutsche Grammatik. Heidelberg: Groos.

ders. (2004): Deutsche Grammatik - Neubearbeitung. München: ludicium.

Figge, Udo L. / Matteis, Mario de (1982): Sprachvergleich Italienisch-Deutsch. 1. Aufl. München: Oldenbourg.

Friederich, Wolf (1994): „Ein größeres Zimmer - a larger room? Zur Wiedergabe absoluter Komparative im Englischen.“Lebende Sprachen 39, 162-163.

Hentschel, Elke / Weydt, Harald (2013): Handbuch der deutschen Grammatik. Berlin: de Gruyter. Kleineidam, Hartmut / Raupach Manfred (1995): „Grammatiken“. Bausch, Karl-Richard / Christ, Herbert / Hüllen, Walther / Krumm, Hans-Jürgen (Hrsg.): Handbuch Fremdsprachenunterricht. Tübingen/Basel: Francke, 298-301.

Jung, Walter (1980): Grammatik der deutschen Sprache. Bearbeitet von Günter Starke. 7., unverändert. Auflage. Leipzig: Bibliogr. Inst.

Nied Curcio, Martina (2008) (Hrsg.): Phänomene zur kontrastiven Linguistik Italienisch-Deutsch. Ein Studien- und Übungsbuch für italienische DaF-Studierende. Milano: Franco Angeli.

Paul, Hermann (1916-1920): Deutsche Grammatik. 5 Bde. Halle: Niemeyer. Nachdruck 1968. Tübingen: Niemeyer.

Renicke, Horst (1955): „Steigerungsinversion.“ Zeitschrift für deutsche Philologie 74, 280-285.

Schefer-Krayenbühl, Ursula (1989): Der absolute Komparativ. Ein Vergleich zwischen dem Englischen und dem Deutschen. Zürich Univ., Diss.

Thurmair, Maria (2001): Vergleiche und Vergleichen. Tübingen: Niemeyer.

Trost, Igor (2006): Das deutsche Adjektiv. Untersuchungen zur Semantik, Komparation, Wortbildung und Syntax. Tübingen: Francke.

Weinrich, Harald (1987). „Ein kürzer Beitrag zu den Norm-Komparativen der deutschen Sprache“. Aust, Gerhard (Hrsg.): Wörter, Schätze, Fugen und Fächer des Wissens. Tübingen: Narr, 115-124. 
Wellmann, Hans (2008): Deutsche Grammatik. Heidelberg: Winter Verlag.

Zifonun, Gisela / Hoffmann, Ludger / Strecker, Bruno (1997): Grammatik der deutschen Sprache. 3 Bde. Berlin: de Gruyter.

\section{Deutsche Fremdsprachengrammatiken für Italiener}

Ahrenholz, Bernt (1994): Grammatica tedesca per principianti. Schena: Fasano.

Alella, Anna Maria / Marini, Ida (1962) (1971): Deutsche Sprachlehre - Grammatica tedesca. Milano: Signorelli.

Azaïs, Alban (1997): Grammatica pratica del tedesco dalla A alla Z. Milano: Hoepli.

Bornmann, Fritz / Mauser, Walther (1960): Corso di lingua tedesca: letture, grammatica, esercizi e vocabolario. Firenze: Ed. Valmartina.

Bruno, Elena / Franch, Raffaella (1991) $\left(1994^{3}\right)$ : Deutsche Grammatik. Grammatica di riferimento per lo studio della lingua tedesca. Torino: Il Capitello.

Cannillo, Luigi / Nasoni, Roberta (1993): Grammatica tedesca con esercizi. Milano: Edizioni Scolastiche Bruno Mondadori.

Difino, Elisabetta / Fornaciari, Paola (1999): Tipps. Milano: Principato.

Dreyer, Hilke / Schmitt, Richard (1993) (1999): Grammatica tedesca con esercizi. Ismaning: Verlag für Deutsch.

Gras Ferraresi, Brigitte / Tomelleri Kromberg, Luciana (1994): Confronti / Vergleiche: Aspetti contrastivi del tedesco rispetto all'italiano. Bologna: Zanichelli.

Laviat, Karin (1996): Deutsche Grammatik. Milano: Spiga Languages.

Luscher, Renate / Schäpers, Roland (1976) (1989): Grammatica del tedesco contemporaneo. Milano: Hueber.

Mittner, Ladislao (1959 ${ }^{10}$ ) (1990): Grammatica della lingua tedesca. Milano: Bruno Mondadori. Motta, Giorgio (2004): Grammatik direkt. Grammatica tedesca con esercizi. Milano: Loescher Editore.

Reimann, Monika (1998): Grammatica di base della lingua tedesca. Ismaning: Hueber.

Saibene, Maria Grazia (1992) (2002): Grammatica descrittiva della lingua tedesca. Roma: La Nuova Italia Scientifica.

Seiffarth, Achim / Medaglia, Cinzia (1998): Arbeitsgrammatik. Rapallo: Cideb.

Schulz, Dora / Griesbach, Heinz (1973) (1988): Deutsche Sprachlehre für Italiener. Grundstufe. Milano: Mursia.

Vanni, Angela (2000): Grundgrammatik Deutsch Italien. Torino/Valmartina/Frankfurt a.M./: Diesterweg.

Weerning, Marion / Mondello, Mariano: (1996) (2000): Dies und Das. Grammatica di tedesco con esercizi. Rapallo: Cideb.

\section{Italienische Fremdsprachengrammatiken für Deutsche}

Blumhaagen, Friedhelm von (1995): Neue italienische Grammatik. 1. Auflage. Göppingen: Das Neue Fachbuch.

ders.: (1997) (2007): Neue italienische Grammatik. Nürtingen: dnf-Verlag. 
Esposito, Maria Antonia / Ressler, Wolfgang (1998) (2003): Langenscheidts praktische Grammatik Italienisch. Berlin/München u. a.: Langenscheidt.

Kirsten, Gerhard / Mack, Barbara (2009): Grammatica italiana per tutti. 2., überarb. Aufl. Stuttgart: Klett Sprachen.

Krenn, Herwig (1996): Italienische Grammatik. 1. Aufl. Hueber: Ismaning.

Mäder, Rolf / Bargagli Stoffi, Barbara (1991): Grammatica d'italiano. Bologna: Il Mulino.

Reumuth, Wolfgang / Winkelmann, Otto (2011): Praktische Grammatik der italienischen Sprache.

Wilhelmsfeld: Egert.

Rovere-Fenati, Beatrice (2013): Praxis-Grammatik Italienisch. Stuttgart: Pons.

Schwarze, Christoph (1978): Kasusgrammatik und Sprachvergleich. Tübingen: TBL-Verlag Narr. ders.: (1988): Grammatik der italienischen Sprache. Tübingen: Max Niemeyer.

\section{Wörterbücher DaF}

Balhar, Susanne (2004): Pons. Großwörterbuch Deutsch als Fremdsprache. Stuttgart et al.: Klett Sprachen.

Cyffka, Andreas (2006): Pons. Großwörterbuch Deutsch als Fremdsprache. Stuttgart et al.: Klett Sprachen.

Dudenredaktion (2002) (2010): Duden. Wörterbuch Deutsch als Fremdsprache. Mannheim/ Leipzig/Wien/Zürich: Dudenverlag.

Kempcke, Günter (2000): Wörterbuch Deutsch als Fremdsprache. Berlin et al.: de Gruyter.

Langenscheidt-Redaktion (1993 und folgende Neubearbeitungen): Langenscheidt Großwörterbuch Deutsch als Fremdsprache. Berlin: Langenscheidt.

Wahrig-Burfeind, Renate (2008): Wahrig. Großwörterbuch DaF. Gütersloh et. al.: BertelsmannLexikon-Inst. et al.

Wolski, Werner (2011): Pons. Großwörterbuch Deutsch als Fremdsprache. Stuttgart et al.: Klett Sprachen.

\section{Wörterbücher des Deutschen und des Italienischen}

Dudenredaktion (1989 und folgende Neubearbeitungen): Deutsch Universalwörterbuch. Mannheim: Dudenverlag.

Wahrig, Gerhard (1996) (2000): Deutsches Wörterbuch. Gütersloh et. al.: Bertelsmann-LexikonInst. et al.

Wahrig-Burfeind, Renate (2006) (2011): Deutsches Wörterbuch. Gütersloh et. al.: Wissen-MediaVerlag.

Zingarelli, Nicola (2010): Vocabolario della lingua italiana. Bologna: Zanichelli. 


\section{Wörterbücher Deutsch/Italienisch Italienisch/Deutsch}

Bidoli, Emilio / Cosciani, Guido (1957): Dizionario italiano-tedesco tedesco-italiano. Torino et. al: Paravia.

Centro Lessicografico Sansoni (1975 und folgende Neubearbeitungen): Dizionario Tedesco Italiano - Italiano Tedesco. Firenze: Ed. Sansoni.

Ciardi Dupré, Giuseppe / Escher, Angelica (1955) (1958): Dizionario italiano-tedesco, tedescoitaliano. Torino: Società editrice internazionale.

Giacoma, Luisa / Kolb, Susanne (2001 und folgende Neubearbeitungen): Dizionario tedescoitaliano italiano-tedesco. Bologna: Zanichelli. 